\title{
Belphégor
}

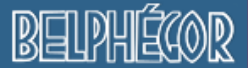

Littérature populaire et culture médiatique

$18-2$ | 2020

Roman Populaire Espagnol - Roman historique, 1900-1950

\section{José Mallorquí, su influencia en la literatura popular}

\section{Ramón Charlo}

\section{(2) OpenEdition \\ Journals}

Electronic version

URL: http://journals.openedition.org/belphegor/3077

DOI: $10.4000 /$ belphegor.3077

ISSN: 1499-7185

Publisher

LPCM

Electronic reference

Ramón Charlo, "José Mallorquí, su influencia en la literatura popular", Belphégor [Online], 18-2 | 2020,

Online since 16 December 2020, connection on 28 April 2021. URL: http://journals.openedition.org/

belphegor/3077 ; DOI: https://doi.org/10.4000/belphegor.3077

This text was automatically generated on 28 April 2021.

\section{(c)}

Belphégor est mis à disposition selon les termes de la Licence Creative Commons Attribution - Pas d'Utilisation Commerciale - Pas de Modification 4.0 International. 


\title{
José Mallorquí, su influencia en la literatura popular
}

\author{
Ramón Charlo
}

\section{Ligeras pinceladas de su vida}

1 José Mallorquí Figuerola nació en Barcelona, el día 12 de febrero de 1913, en una casa de la calle Hospital. Su madre era soltera, por lo que fue inscrito con los dos apellidos de ésta, se llamaba Eulalia y su padre, José Serra Farré, estaba casado con otra mujer, era una persona rica y de cierta importancia en el mundo de la hostelería. Cuando se quedó viudo ya no existían los lazos afectivos ni la atracción de quince años antes, por lo que aunque hubo un acercamiento importante, no se decidió a abandonar la viudedad. Eulalia al no conseguir el matrimonio intentó que le diera sus apellidos al hijo de ambos, es decir que lo reconociese legalmente. Todavía es difícil de comprender por qué no lo hizo, ya que no tenía descendientes, su única hija, hermana de Mallorquí por lo tanto, había muerto años antes. Una justificación plausible podría estar en el poco aprecio que le demostraba su hijo, y en el nulo cariño que había entre los dos.

2 El hecho de ser hijo natural marcó a José Mallorquí en su niñez, e incluso en su primera juventud, haciéndolo retraído, sin amigos, siempre temeroso que otros niños le echaran en cara la carencia de padre, (cosa totalmente incierta pues padre siempre se tiene), sin interés por nada y menos por los estudios. Se crió junto a una amiga de la madre, a la que siempre recordó, ya que, falto del cariño de los padres que veía tenían otros niños, se aferró a su abuela, como él la llamaba. Después de peregrinar por unos cuantos colegios, en los que no aprendió nada, aterrizó en la Escuela Francesa, en la que aprendió francés y poco más. A los once años se despertó en él un afán por la lectura desmedido. En efecto, descubrió a Alejandro Dumas, y no se apartó de él hasta que no acabó con todas sus obras, algunas en español, al principio, y luego, aprovechando su conocimiento del francés, todas en su idioma original.

3 A esto le siguió una etapa, mientras se empleaba en trabajos de poquísima importancia, en la que se dedicó a leer sin freno ni orden, cualquier libro era bueno y cualquier autor 
apropiado: Zane Grey, Alarcón, Galdós, Zola, Blasco Ibáñez, Oliver Curwood, Jardiel Poncela..., con lo que la poca cultura y conocimientos adquiridos en el tiempo de estudiante se completó increíblemente.

4 En esta época compró en una librería de libros usados un ejemplar que debía de influir poderosamente en toda su vida como escritor. Nos referimos a una edición de Los exploradores españoles del siglo XVI, de Charles F. Lummis. Obra escrita por un norteamericano como homenaje a un puñado de españoles. El citado volumen llevaba una fotografía del autor con esta dedicatoria: “¡Viva España. Madre de América!”. Durante mucho tiempo José Mallorquí se hizo varias preguntas: ¿Cómo no se daba mayor difusión a estos hechos que superaban claramente a toda la fantasía de sus autores predilectos?; ¿Por qué no había libros sobre las gestas de los conquistadores y aventureros españoles en América?; ¿A que era debido que no se novelasen estas vidas y esos hechos? Probablemente años después José Mallorquí quiso, si no dar respuesta a ellas, sí crear un héroe que descendiendo de uno de estos hombres conservaba el orgullo y los valores de aquella raza, a la que, desgraciadamente, podemos considerar desaparecida en estos tiempos.

En el año 1931 muere su madre, con la que él vivía desde que salió del internado de Sarriá, y a la que indudablemente había dado parte del cariño que se tiene a una madre, quedando solo a la temprana edad de dieciocho años. El padre inició un tímido acercamiento que fue rechazado por Mallorquí, que seguía sin poder soportarlo y que lo trataba muy fríamente, lo que conllevó a la ruptura total, sobre todo cuando José Mallorquí se despidió del trabajo y se dedicó a lo que algunos dirían que a vivir, pero en realidad lo que hizo fue malgastar la herencia que le había dejado su madre, a la que se sumó una jugosa cantidad que José Serra Farré había dado para su hijo cuando decidió separar su vida de la de Eulalia. Claro está el dinero no dura eternamente, por lo que al cabo de cierto tiempo se encontró con que no tenía un duro y había que seguir viviendo. Ahora bien durante éstos dos años había hecho amigos, nada más fácil teniendo dinero; practicado deportes, nada más lógico teniendo mucho tiempo libre; $\mathrm{y}$, sobre todo, se había enamorado, nada más factible siendo joven. En efecto, había conocido a una chica algo mayor que él, con la que se sentía seguro y, lo más importante, se veía valorado y querido casi por primera vez. Por ello no es de extrañar que tomara la decisión de que Leonor del Corral, que así se llamaba, fuera la mujer de su vida, formalizando relaciones. Por estas fechas se presentó a la editorial Molino que necesitaba traductores, corría el año 1934, y pensaba que las obras a traducir serían francesas, idioma que dominaba, pero no fue así, ya que eran inglesas, estuvo a punto de abandonar, pero como no encontraba otra salida y con el estímulo de Leonor, su novia en aquel entones, estímulo que no le faltó en ningún momento mientras vivió, siguió adelante. Las traducciones de José Mallorquí resultaron magníficas, era un traductor literario, utilizado el termino en contraposición de literal, es decir que interpretaba lo que quería expresar el autor y así lo reflejaba. En cierto modo era un creador de la obra en otro idioma. Eso le permite casarse, a poco de empezar la tristísima Guerra Civil española, terminada esta sigue traduciendo y empieza a escribir relatos cortos y novelas deportivas, algunos bosquejados en los duros años de la guerra, que publicaba la editorial Molino. Transcurrido un año, es decir en 1940, nace su primer hijo, Juan Carlos. A pesar de las frías y casi nulas relaciones de José Mallorquí con su padre, fue el elegido por Leonor como padrino del niño, intentando un acercamiento y buscando, sin duda, que lo nombrase heredero, ya que no tenía otros descendientes, a pesar de que José Mallorquí seguía sin tenerle la más mínima simpatía, ya que cariño o 
amor filial nunca existió entre ellos. Las mujeres siempre han sido más practicas que los hombres, pero en éste caso la cosa no resultó, ya que al morir el abuelo, unos años después, se olvidó totalmente de él.

6 Poco a poco José Mallorquí se va abriendo paso en el dificilísimo mundo de las letras, y deja de ser traductor para pasar a ser escritor. Sus inicios fueron duros, ya que, excepto su mujer, nadie confiaba en sus dotes. Y le llega el éxito y el reconocimiento con la colección El Coyote. Sus relatos son seguidos y leídos con avidez no sólo en España, ya que se publican en diferentes países de Europa y en Hispanoamérica. Entretanto había nacido su segundo hijo, al que le ponen el nombre de Eduardo, y años después, en 1953, el tercero y último, que recibe el nombre de César.

7 Pero en esta vida todo tiene un final, y, a principios de 1954, José Mallorquí decidió abandonar la novela popular. Sus innegables dotes de creador hacían que no le faltaran oportunidades para ello y, después de una breve incursión en el mundo del cine, acepta una oferta de Manuel Aznar, directivo de la Sociedad Española de Radiodifusión (Cadena SER), y empieza a trabajar en la radio, aportando la frescura de sus ideas y sus fantásticas dotes de escritor, que le llevaron a conseguir importantes galardones, entre los cuales podemos citar los Premios Ondas de los años 1954 y 1964, y el Premio Nacional de Radio de 1965.

8 La enfermedad y muerte de su esposa, ocurrida, en el año 1971, le afecta profundamente, y, desde entonces, José Mallorquí no fue el mismo, falto de su apoyo, la inseguridad de sus primeros años se apoderó de nuevo de él, y con un disparo puso un fin violento a su vida.

9 Para finalizar esta semblanza me gustaría incluir una breve descripción física de Mallorquí, y nada mejor que la que hace su hijo César:

Mi padre tenía un aspecto físico muy peculiar. Lo primero que llamaba la atención era su gordura, una gordura tensa, en ningún caso fofa, que se manifestaba exclusivamente en un abultado estómago de proporciones pantagruélicas. Sin embargo, sus piernas y sus brazos eran delgados y poseía un buen tono muscular. En su caso, la grasa jamás se acumuló en la sotabarba ni en ninguna otra parte de su cuerpo. Por decirlo con sencillez: José Mallorquí tenía una tripa enorme, pero, por lo demás, era delgado. No puede decirse que fuese un hombre alto, medía un metro setenta, pero sí fornido, aunque un poco cargado de espaldas (supongo de tanto permanecer inclinado sobre la máquina de escribir). Calvo desde muy joven, su cráneo era redondo y compacto, semejante a la cabeza de un bulldog. Gafas de miope, gruesas como culos de botella, cabalgaban sobre una nariz más bien chata, convirtiendo los ojos de Mallorquí en dos puntos muy intensos parapetados tras sendas murallas de cristal. Su mirada era peculiar: en menos de un segundo pasaba de la extrema seriedad a una alegre picardía, del ensimismamiento más profundo a la franca extroversión... ¿Qué más decir del aspecto físico? Ah, claro, el bigote. Le acompañó durante tantos años que acabó convirtiéndose en un rasgo clave de su fisonomía". ("Los ojos de José Mallorquí. Revista del Circulo Andaluz de Tebeos", n. 19).

\section{Sus inicios y su evolución}

10 Sus inicios fueron duros, ya que, excepto su mujer, nadie confiaba en sus dotes. Así vemos que su primera novela $\mathrm{El}$ primer fracaso del comisario Martínez, fue rechazada por cuantos editores la tuvieron en sus manos. Dos meses escasos después de este revés termina su segunda novela, El despertar de Cenicienta, que es aceptada por la editorial 
Molino para su colección Violeta. El beneficio material que le dio fue más bien descorazonador, escasamente mil pesetas, algo más de trescientas pesetas que una traducción. Si no llega a ser por el ánimo de su mujer hubiera abandonado, pero esta confianza, que nunca le faltó, hizo que siguiera escribiendo y rápidamente terminó $\mathrm{El}$ misterio de los guantes negros y El misterio de los tres suicidas, que fueron aceptadas por Molino para su Biblioteca Oro. A esto hay que añadir una serie de relatos que se publicaron en una colección argentina titulada La Novela Deportiva, en los que las protagonistas se mueven en el alegre ambiente universitario americano. Por lo tanto estamos hablando de deporte aficionado (realizado por no profesionales), el deporte por el deporte o el deporte por la gloria, aunque, dado su número, es lógico que encontremos algunos en que los protagonistas son profesionales, principalmente las que giran en torno al mundo del boxeo o del ciclismo. También de esta época son alguna que otra novela policíaca o de aventuras en series de la editorial Molino.

11 Pero cuando José Mallorquí empieza a tener cierto nombre y a ser conocido es cuando en 1943 la Editorial Clíper decide lanzar una colección de novelas populares del 'Oeste americano' ya que escoge como escritor principal a José Mallorquí, como se desprende que de los primeros cincuenta y un títulos, incluidos los extras, treinta y tres fueran de este autor. Al principio, temeroso Germán Plaza de que no tuviesen buena acogida entre los lectores, dado el poco prestigio de los autores españoles, decide que estos aparezcan con seudónimo de fonética extranjera, por lo que las primeras novelas de Mallorquí aparecen con los seudónimos de Carter Mulford, Leland R. Kitchell o E Mallory Fegurson, aunque al poco tiempo la administración española consideró que estas practicas podían ser un fraude y obligó a abandonarlas. De esta época es una de las mejores novelas escritas por él, La venganza de "Amarguras", (para algunos la mejor).

\section{El mundo de su "oeste". Su estilo y su influencia en otros escritores}

Un aspecto muy importante cuando se estudia la obra de Mallorquí es examinar cómo es el "oeste" que nos presenta. Ya que no es el tradicional oeste salvaje, repleto de pistoleros, vaqueros, "saloons", e indios feroces y brutales, propios de la colonización de territorios indios y grandes llanuras semidespobladas. Mallorquí nos presenta a una California que ya estaba colonizada por los españoles y en ella existía una cultura en muchos aspectos bastante superior a la de los norteamericanos, sobre todo de los que solían aparecer por allí: duros, violentos, de las capas más bajas de la organización social, sobre todo en sus países de origen, en los que soportaban la pobreza envidiando a otros que lo tenían todo, que se embarcaban en una aventura arriesgada hacia unos territorios alejados y llenos de peligro con el único objetivo de conseguir unas mejores condiciones de vida para ellos y su familia, y unas tierras, en cuya posesión la mayoría cifraba la riqueza, que fueran de su propiedad. Emigrantes en busca de la "tierra de promisión", deseada por su clima, fertilidad del suelo y las riqueza que se podían conseguir, principalmente oro. Esto no quiere decir que no aparezcan pistoleros, indios, ladrones de bancos, asaltantes de diligencias, etc., ya que en alguna medida tienen que existir en las obras de este "género del oeste".

Por las novelas del oeste de Mallorquí desfilan una gran variedad de personajes: caballeros, unos que hacen honor a este apelativo y otros que sólo podrían recibir ese calificativo cuando montan a caballo; políticos corruptos cuyo interés no es el de la 
comunidad sino exclusivamente el suyo propio, que incluso falsean datos de los ayuntamientos que rigen para quedarse con el dinero de las subvenciones y sueldos que pagan; rapaces hombres de negocios que aprovechan la construcción de una línea ferroviaria para, mediante la extorsión y la amenaza, engrosar sus cuentas corrientes; falsos patriotas cuya única patria es la riqueza personal; médicos rurales que son capaces de atender a enfermos necesitados sin cobrar y a exponer su vida para defender sus ideales; espías femeninos que a su belleza unen una inteligencia y clarividencia muy fuera de lo común; idealistas y soñadores que viven anclados en el pasado y aspiran a que este vuelva, sin comprender que el agua que pasa bajo un puente nunca vuelve a pasar otra vez por él; militares diversos, unos que piensan que para escalar el escalafón todo está permitido y otros que hacen culto al honor, al valor y la honradez; viejos hidalgos descendientes de españoles para los que cualquier tiempo pasado fue mejor, incapaces de comprender la evolución de los antiguos territorios pertenecientes a Nueva España; frailes franciscanos que, apartados de la labor que hacían en las misiones por la llamada "ley de amortización", mal viven ahora en las pocas instituciones que les han dejado intentando ayudar a los más desfavorecidos; risueños contrabandistas de armas que de su profesión hacen un arte; aventureras capaces de los mayores sacrificios para hacer olvidar su turbio pasado...

Otro punto que hay que resaltar es que cuando Mallorquí empezó a escribir novelas populares el mercado español de este tipo de obras estaba siendo acaparado por traducciones, por lo que el escritor español del género se veía en la tesitura de imitar a estas, o crear una autentica y diferenciada novela española. El primero era un camino fácil y que además Mallorquí dominaba, en gran parte debido a las múltiples traducciones que había realizado para la editorial Molino, pero no fue el que escogió, optó por lo que los estudiosos han denominado "la españolización de la novela del Oeste". El lugar es el mismo, el oeste de Norteamérica, el tiempo es el mismo, los años de la conquista y colonización (1840-1890), y los héroes, aunque lógicamente, son también norteamericanos, a casi todos ellos se les dota de un profundo sabor español.

En efecto, con respecto al lugar, Mallorquí maneja el hecho histórico que una parte muy importante de lo que se ha llamado "Oeste" es originariamente español, ya que Tejas, California y Nuevo Méjico habían formado parte del Virreinato de Nueva España. En estos territorios los españoles, o descendientes de estos, fueron sus primeros pobladores y los que colocaron las bases fundamentales de su civilización y cultura. Por lo tanto es un "oeste" más español que norteamericano. Si nos fijamos en los personajes que intervienen en sus historias a pesar de que son norteamericanos, como es lógico si las aventuras tienen lugar en Norteamérica, los que tienen protagonismo son, además, descendientes de españoles, hispanoparlantes que se enorgullecen de su ascendencia y de su historia.

16 Muchos autores intentaron copiar este estilo, pero este modelo era difícil de adoptar en las novelas del "oeste", ya que, para la mayoría de los autores, la acción es lo único importante, y sus personajes eran siempre los mismos, cuatro o cinco tipos que aparecen una y otra vez y que actúan bajo unos patrones prefijados. Sin embargo, la cosa cambiaba en las novelas de aventuras y así vemos, por ejemplo, a Arnaldo Visconti, que en colecciones tales como El Pirata Negro, El Galante Aventurero o Diego Montes, sus héroes son españoles; a A. Cienfuegos, que Capa Roja, el héroes de la colección Espadachín, aunque nacido en Bélgica es de padre español y hace alarde de ello; a J. 
León, que en su colección El Corsario Azul, la gran mayoría de los personajes son españoles...

Por último debemos resaltar que la prosa que utiliza Mallorquí es sobria y sencilla, nunca recurre a querer adornarse con bellas frases, ni imágenes complicadas, impropias de este tipo de literatura, destacando también la pureza del español que utiliza, su urbanidad gramatical, la regularidad de su sintaxis y la exactitud de los términos que emplea.

\section{El éxito de la colección El Coyote en España}

18 El éxito obtenido por la colección El Coyote nunca ha sido superado por alguna que otra colección de este tipo. Empezó, en septiembre de 1944, con una tirada de 10.000 ejemplares y su primer número se agotó en un par de días, por lo que hubo que hacer otra tirada de casi el mismo número de ejemplares, que también se agotó rápidamente. Esto hizo que número a número se superara la tirada estabilizándose en alrededor de 50.000 ejemplares (aunque en la época de su máximo esplendor la tirada era de 65.000). Luego el número fue descendiendo lentamente $\mathrm{y}$, a pesar de que cada volumen se mantuvo en un precio bajo y que se cambió el formato, el mercado daba muestras de cansancio por lo que las tiradas rondaban los 12.000 ejemplares. Finalmente, por diversas causas, entre las que la principal era la desavenencia con el editor, la colección se interrumpió bruscamente para ya nunca reanudarse.

¿Por qué tuvo tanto éxito la colección El Coyote? Hay varias razones, la calidad literaria de los relatos no es, por supuesto, ajena a ello, pero para mí la razón fundamental fue una consecuencia directa de eso. En efecto, los potenciales lectores se multiplicaron, por lo que sus novelas, contrariamente a la mayoría de las obras de este tipo, podían gustar, como así fue, a personas que tuviesen cierta formación y que requiriesen que los relatos tuviesen cierta calidad. Sin dudar, yo me atrevería a decir que para comprender las novelas de Mallorquí hace falta tener un cierto conocimiento de la historia, tanto de la norteamericana: la Guerra de la Independencia Americana, la colonización del Oeste, la guerra contra Méjico, la guerra de la Secesión, las guerras indias, el descubrimiento de oro en un molino de Sutter, etc., etc.; como de la española: el descubrimiento y colonización de América, la existencia y funcionamiento de los Virreinatos, la colonización espiritual de California por los franciscanos, los diferentes procesos de independencia de los estados iberoamericanos, etc., etc., ya que son elementos que el autor utiliza y referencia continuamente en sus novelas, mezclándolos de forma inapreciable con otros hechos salidos de su imaginación.

\section{La colección El Coyote en otros países}

El enorme éxito que alcanzaba en España las aventuras del famoso enmascarado californiano hizo que prácticamente en todos los países de Europa se publicaran colecciones de El Coyote, no teniendo en todos ellos la misma acogida. 


\section{Alemania}

21 En este país El Coyote se publicó de forma totalmente desordenada. En efecto, se lanzaron al mercado cuatro colecciones diferentes, aunque ninguna fuera completa y tampoco entre todas se completara entre todas ellas. Solamente la primera tuvo cierta importancia, pues las otras, por el número de títulos que sacaron, fueron meramente testimoniales.

La primera empezó a publicarse en 1949, saliendo solamente 74 números a lo largo de seis años. Por lo tanto, el último salió en 1955. Su título genérico era Coyote, y, probablemente, para diferenciarla de la edición austriaca se añadía Deutshe Ausgabe. La colección tuvo muchísimo éxito en sus inicios, con tiradas superiores a los 70.000 ejemplares. A partir del número 30, coincidiendo con el hecho de que se cambiase de editorial (hasta entonces era Werner Nowack, amigo personal de Germán Plaza, lo que hace que la colaboración de este casi desaparezca), las tiradas, que ya un poco antes habían iniciado un ligero declive, bajan casi a la mitad, entre otras cosas por aburrimiento de los lectores, ya que el tiempo que transcurre entre la salida de un número y el siguiente es, normalmente, excesivo, y, poco a poco la colección languidece hasta desaparecer.

Un caso sorprendente es el número 74 ya que no corresponde con ninguna novela escrita por Mallorquí, por lo tanto estamos ante una novela apócrifa, que se hizo para poner un fin a la colección.

En la segunda salieron solamente 7 números, teniendo cada uno de ellos dos títulos.

En la tercera salieron 20 títulos, y fue una muestra clara del desorden que imperó en la publicación. El tiempo del enmascarado ya había pasado en Alemania y no tuvo buena acogida, las tiradas no superaron los 3.500 ejemplares en ningún momento, por lo que no era rentable, a pesar de que se procuró que los títulos que se incluyeran no se hubieran publicado anteriormente en el país germano.

\section{Austria}

Tampoco en Austria se publica de forma completa, pues la editorial Schwicker-Linz publicó 43 títulos. La colección empezó con unas tiradas muy sugestivas, muy similares a las que tenía la edición alemana, pero pasó una cosa bastante curiosa, los austriacos preferían comprar la edición alemana, a pesar de que eran bastante parecidas, lo que hizo que las ventas de esta publicación bajaran drásticamente, lo que la llevó a su interrupción.

A poco de terminar la anterior, ya en 1953, la editorial Pelda-Wien continúo con la publicación, a partir de donde la dejó Schwicker-Linz, sacando diez títulos más, con la particularidad de que no estaban agrupados en una colección titulada El Coyote.

\section{Dinamarca}

En este país dos editoriales publicaron números de la colección El Coyote, ninguna de ellas completa. La primera, Nyt Dansk Forlag - Kobenavn, empezó en el año 1954. La colección tuvo al principio bastante éxito, pero poco a poco los lectores fueron perdiendo interés, cosa por otra parte comprensible ya que no se puede mantener este 
sacando al mercado 46 números en algo más de cuatro años, no saliendo de media ni un número al mes.

En el año 1971 la Winthers Forlag/Interpresse se lanzó a la aventura de publicar de nuevo la colección El Coyote, su éxito fue escaso, los lectores ya habían olvidado al héroe enmascarado y no se interesaron, en absoluto, por la nueva edición, por lo que pronto se dejó de publicar, solamente salieron al mercado ocho números.

\section{Finlandia}

La colección El Coyote fue publicada también en Finlandia, aunque como en casi todos los países de habla no hispana no llegó a salir completa. Se hicieron dos intentos. En 1953 la editorial Kustannusliike Sarjakirja-Helsinki lanzó al mercado una colección que tituló El Coyote. Solamente salieron 78 números, siendo el formato exactamente igual que el de la colección española original publicada por Clíper, es decir 21 x $15 \mathrm{~cm}$.

31 Diecinueve años después, es decir en 1989, la editorial Jalava-Helsinki publicó otro volumen que llevaba cuatro novelas.

\section{Francia}

Francia fue uno de los países europeos donde la colección El Coyote tuvo menos éxito, aunque en un artículo firmado por Luis Montañés Fontela, que apareció en la Bibliografía Hispánica correspondiente al mes de enero de 1950, se dice, al hablar de la difusión de El Coyote, que la tirada en Francia era de 60.000 ejemplares, yo no comparto dicha aseveración, pues en ese caso no hubiese dejado de publicarse cuando habían salido solamente catorce títulos. La editorial era Editions Librairie Moderne de París y la traducción estuvo a cargo de Monique George.

\section{Inglaterra}

La colección protagonizada por el famoso enmascarado californiano empezó a publicarse en lengua inglesa en 1951, siendo su editor Gaywood Press, London. Como no podía ser de otra forma se tituló The Coyote y tuvo una corta vida, pues su acogida no fue buena, cosa completamente lógica, ya que los ingleses no podían valorar bien una serie en la que el protagonista era español, los co-protagonista de habla española y los malvados de habla inglesa. Salieron únicamente 12 títulos, todos a lo largo del año señalado, siendo la traducción de Peter Audsley.

\section{Italia}

Este país fue donde más aceptación tuvieron las novelas escritas por José Mallorquí (claro está que sin contar a España) y también fue de los primeros en los que se empezaron a publicar.

A pesar de esto, en Italia no se hizo una edición que pueda servir de ejemplo del bien hacer, ya que intentos hubo varios pero sus resultados no fueron todo lo bueno que merecían, tanto el autor como los lectores. Que conozcamos entre el año 1947 y 1958 se realizaron ocho intentos, en ninguno de ellos aparecieron todos los títulos, aunque entre todos se completara la colección. De estos el que merece recordarse es la llamada 
Collana El Coyote, que salió en dos etapas, en la primera, que empezó en el año 1950, salieron 24 números, y la segunda, febrero de 1951, reanuda la publicación de la colección, y aunque lo hace a partir del número último publicado en la anterior, la numeración de los volúmenes comienza otra vez desde el 1. Los volúmenes llevaban además de la novela correspondiente del Coyote, otra serie de cosas. Así nos encontramos artículos divulgativos, páginas de pasatiempos, pequeños relatos de diversos tipos, e incluso novelas del oeste, publicadas originalmente en España en la colección Novelas de Oeste (editorial Clíper), que aparecían parcialmente en números correlativos.

Esta colección tuvo un gran éxito, alcanzando grandes tiradas, tanto es así que la quisieron prolongar incluyendo otras que, aunque eran de José Mallorquí, no tenían como personaje central al Coyote, pero la cosa no funcionó, sobre todo con las últimas novelas provenientes de Dos Hombres Buenos, lo que hizo que se decidiera no seguir con la publicación de estas.

\section{Noruega}

37 En este país se empezó a publicar en el año 1952, no completándose, pues salieron únicamente cuarenta y siete títulos, a lo largo de más de cuatro años, ya que el último vio la luz en 1957. El formato de los libros era un tanto intermedio entre el clásico bolsilibro y el que llevó la edición original española de clíper, ya que sus medidas eran de 18 x 12,5 cm. La colección tuvo muy buena acogida y sus ventas, al principio, fueron más que interesantes, pero el público se cansa y pierde la costumbre de seguir el personaje si sale una media de una novela cada mes.

\section{Portugal}

Portugal, junto a Francia e Inglaterra, fueron los países europeos donde la colección $E l$ Coyote tuvo menos éxito. En efecto, se inició únicamente una edición, publicándose solamente diez números, siendo la editorial IBIS, la periodicidad era mensual, demasiado tiempo esperando los potenciales lectores para adquirir el siguiente título, hecho al que yo le achaco una parte del fracaso y no, como dicen alguno, a que en Portugal se vendieron los números publicados en Brasil, aprovechando el que compartían el idioma, pues esto no me consta y si se hizo sería de forma bastante testimonial.

\section{Suecia}

La casa editorial Lindqvists-Stockholm comenzó, en el año 1954, la aventura de la publicación de la colección. Aunque empezó siguiendo el orden de la edición original española, muy pronto lo altera. Publica solamente 46 títulos. La colección tuvo muchos altibajos ya que salía un máximo de nueve números al año. Terminó en el año 1960.

En el año 1971 la casa editorial Semic-Stockholm hizo un nuevo intento para dar a conocer la colección El Coyote, su éxito fue escaso, ya que solamente salieron al mercado seis números. 


\section{Checoslovaquia}

41 Existe cierta controversia sobre la existencia o no de una edición checa, yo pienso que no, ya que no he encontrado referencia alguna ni en los archivos de la Biblioteca Nacional Checa, ni en mis búsquedas de algún ejemplar por internet. Sin embargo, he encontrado dos menciones al respecto. La primera en la obra La novela popular en España: José Mallorquí, de Juan Francisco Álvarez Macías, en la que da una relación de los países en donde se publicaron sus aventuras, incluyendo a Checoslovaquia, especificando que hasta que el golpe comunista la hizo desaparecer, pero no añade ni un solo dato al respecto. La segunda mención que he encontrado ha sido en el artículo "Análisis de un sorprendente éxito editorial”, firmado por Luis Montañés Fontenla, que se publicó en la Bibliografía Hispánica en febrero de 1950, que dice: “El Coyote comenzó a ser pedido desde América, hasta que se hizo imprescindible la edición americana. En Europa fue solicitado desde Checoslovaquia, donde estuvo muy a punto de aparecer..." Por lo tanto, hay que llegar a la conclusión... que estuvo a punto pero no se hizo.

Fuera de Europa nos encontramos con dos países más en los que se publicaron las novelas de El Coyote, además con gran éxito.

\section{Argentina}

En este país se empezó a publicar la colección El Coyote en el año 1948, en concreto el primer número salió el 26 de Julio de ese año, siendo su editora la misma que en España: Clíper. Pero ya en el segundo la publicación pasa a Queromon Editores, Buenos Aires, que sigue con ella hasta el número 80 , aunque se publicaron un total de 84 , ya que los números $12,13,20$ y 32 se duplicaron. La dirección que aparecía como referencia para ambas editoriales era la misma: Oro 2455 - Buenos Aires. La colección tuvo una muy buena acogida teniendo unas tiradas medias de 50.000 ejemplares, no entendiéndose muy bien por qué dejó de publicarse, pues aunque sus tiradas habían bajado bastante todavía parecía que su publicación era rentable. La única explicación que se me ocurre es que surgieran discrepancias entre las casas editoriales.

\section{Brasil}

Quizás fuese en Brasil en donde la colección El Coyote se publicase de forma más completa y racional, a lo que contribuyó, indudablemente, al enorme éxito que tuvo. En concreto se hicieron cuatro ediciones, la primera la publicó la Editora Monterrey Limitada, la segunda la empezó Bruguera y la continuó Cedibra, siendo la tercera de Zip y la cuarta también de Cedibra, ahora bien solamente la primera y, como mucho, la segunda, merecen el nombre de colección pues las otras dos son meramente testimoniales.

Aunque Francisco Álvarez Macías, en la obra anteriormente citada, nos dice que se hicieron ediciones en Méjico y Chile, yo tengo series dudas, ya que nunca he encontrado un ejemplar de dichas publicaciones, ni tampoco referencia alguna fuera sobre ellas.

Bien, aquí termino este artículo, que espero y deseo que contribuya a conocer mejor al gran escritor que fue José Mallorquí, que engrandeció y dignificó la llamada novela popular, pues con él alcanzó unas cotas que para si quisieran otros tipos de literatura, 
mucho más valorados por algunos críticos, la mayoría de las veces por que no se han molestado en leer las obras de autores de su talla, (o no las han considerado dignas de su tiempo).

\section{ABSTRACTS}

El artículo proporciona una síntesis biográfica de José Mallorquí subrayando los acontecimientos personales que pudieron influir en su trayectoria literaria. En un segundo tiempo se analizan los inicios de Mallorquí en el panorama editorial y cómo se convierte en autor de renombre gracias a la práctica de un género: la novela del oeste, que él adapta a los gustos de su público siguiendo, por otra parte, la moda de su tiempo, amante de imitar las traducciones de novelas extranjeras. Por último se observan las circunstancias que propiciaron el éxito indiscutible de la colección El Coyote tanto en España como en países de toda Europa e incluso de América del Sur.

\section{AUTHOR}

\section{RAMÓN CHARLO}

Investigador

rcharlo@ono.com 